\title{
On the Evaluation of the Proportionality Coefficient between the Turbulence Temperature Spectrum and Structure Parameter
}

\author{
JEREMY A. GIBBS \\ Cooperative Institute for Mesoscale Meteorological Studies, University of Oklahoma, and NOAA/OAR/National Severe \\ Storms Laboratory, Norman, Oklahoma \\ EVGENI FEDOROVICH \\ School of Meteorology, University of Oklahoma, Norman, Oklahoma
}

(Manuscript received 16 December 2019, in final form 27 April 2020)

ABSTRACT

\begin{abstract}
The turbulence temperature spectrum and structure parameter are related through a widely adopted proportionality coefficient. We formally derive this expression, and present further evidence, to demonstrate that this coefficient is too large by a factor of 2 .
\end{abstract}

\section{Introduction}

It is customary to quantify the intensity of turbulent fluctuations of meteorological fields through a single parameter called the structure parameter. The structure parameter, which is assumed constant within the inertial subrange of turbulence scales, is relevant to many applications associated with atmospheric boundary layer processes. One of the most studied structure parameters is the (potential) temperature parameter, which is frequently used to describe properties of electromagnetic and acoustic wave propagation in the atmosphere. A relationship between the scalar structure parameter and the scalar spectral density in the inertial subrange was first obtained by Tatarskii (1961) and evaluated for temperature by Wyngaard et al. (1971). The latter form has been widely used in the engineering and atmospheric sciences for nearly fifty years. We formally rederive this relationship and present evidence to demonstrate that the associated proportionality coefficient is too large by a factor of 2.

\section{Deriving the integral spectral approximation of the structure parameter}

The second-order temperature structure function (Tatarskii 1961; Wyngaard 2010) is defined as

Corresponding author: Jeremy A. Gibbs, jeremy.gibbs@noaa.gov

$$
D_{T}(r)=\overline{[T(x)-T(x+r)]^{2}},
$$

where $T$ is temperature or potential temperature when Eq. (1) is used in the atmospheric context, $x$ is the coordinate direction in space, and $r=|\mathbf{r}|$ is the separation distance (equal to the magnitude of the separation vector). If we assume that turbulence is locally isotropic and that the separation distance lies within the inertial subrange of spatial scales of turbulent temperature fluctuations, the temperature structure function may be expressed as (Kolmogorov 1941)

$$
D_{T}(r)=C_{T}^{2} r^{2 / 3}
$$

where $C_{T}^{2}$ is the temperature structure-function parameter, often just called the temperature structure parameter. The following relationship between $D_{T}$ and the one-dimensional spectral density of temperature fluctuations $\Phi_{T}$ is valid under the assumption of turbulence isotropy (Wyngaard 2010):

$$
\begin{aligned}
D_{T} & =2 \int_{-\infty}^{\infty}[1-\cos (k r)] \Phi_{T}(k) d k \\
& =4 \int_{0}^{\infty}[1-\cos (k r)] \Phi_{T}(k) d k,
\end{aligned}
$$

where $k$ is the wavenumber associated with the $x$ direction. Both Eqs. (2) and (3) are based on the fundamental definition of the structure function and require no additional 
assumptions beyond their theoretical underpinnings. As an aside, Essenwanger and Reiter [1969, their Eq. (6)]—an oft-cited study focused on structure functions and power spectra of atmospheric velocity increments-incorrectly omits the leading factor of 2 in Eq. (3).

One can evaluate the integral in Eq. (3) analytically by assuming that the entire spectrum has the inertial subrange form

$$
\Phi_{T}=A k^{-5 / 3} .
$$

To do this, we substitute Eq. (4) into Eq. (3) to obtain

$$
D_{T}=4 A \int_{0}^{\infty}[1-\cos (k r)] k^{-5 / 3} d k
$$

We next take the integral employing integration by parts:

$$
\begin{aligned}
\int_{0}^{\infty}[1-\cos (k r)] k^{-5 / 3} d k= & \underbrace{-\left.\frac{3}{2}[1-\cos (k r)] k^{-2 / 3}\right|_{0} ^{\infty}}_{=0} \\
& +\frac{3}{2} r \int_{0}^{\infty} \sin (k r) k^{-2 / 3} d k .
\end{aligned}
$$

We use the following table integral from Gradshteyn and Ryzhik [2014, their Eq. (3.761), p. 440] for the remaining part on the right-hand side of Eq. (6):

$$
\int_{0}^{\infty} x^{\mu-1} \sin (a x) d x=\frac{\pi}{2 a^{\mu} \Gamma(1-\mu) \cos \left(\frac{\mu \pi}{2}\right)},
$$

where $x=k, \mu=1 / 3$ according to Eq. (6), and $a=r$. This yields

$$
\begin{aligned}
\frac{3}{2} r \int_{0}^{\infty} \sin (k r) k^{-2 / 3} d k & =\frac{3}{2} r \frac{1}{2 r^{1 / 3} \Gamma\left(\frac{2}{3}\right) \cos \left(\frac{\pi}{6}\right)} \\
& =\frac{3 \pi r^{2 / 3}}{4 \Gamma\left(\frac{2}{3}\right) \cos \left(\frac{\pi}{6}\right)}
\end{aligned}
$$

Noting that $\cos (\pi / 6)=\sin (\pi / 3)$ and $\Gamma(2 / 3)=(3 / 2) \Gamma(5 / 3)$, we have

$\frac{3}{2} r \int_{0}^{\infty} \sin (k r) k^{-2 / 3} d k=\frac{3 \pi r^{2 / 3}}{4 \frac{3}{2} \Gamma\left(\frac{5}{3}\right) \sin \left(\frac{\pi}{3}\right)}=\frac{\pi r^{2 / 3}}{2 \Gamma\left(\frac{5}{3}\right) \sin \left(\frac{\pi}{3}\right)}$.

Thus,

$$
\int_{0}^{\infty}[1-\cos (k r)] k^{-5 / 3} d k=\frac{\pi r^{2 / 3}}{2 \Gamma\left(\frac{5}{3}\right) \sin \left(\frac{\pi}{3}\right)}
$$

and

$$
D_{T}=4 A \int_{0}^{\infty}[1-\cos (k r)] k^{-5 / 3} d k=\frac{2 \pi A r^{2 / 3}}{\Gamma\left(\frac{5}{3}\right) \sin \left(\frac{\pi}{3}\right)} .
$$

Making use of Eq. (2) gives us

$$
\frac{2 \pi A r^{2 / 3}}{\Gamma\left(\frac{5}{3}\right) \sin \left(\frac{\pi}{3}\right)}=C_{T}^{2} r^{2 / 3} .
$$

Solving for $A$ provides

$$
A=\frac{C_{T}^{2} \Gamma\left(\frac{5}{3}\right) \sin \left(\frac{\pi}{3}\right)}{2 \pi} \approx 0.125 C_{T}^{2},
$$

which results in

$$
\Phi_{T}=A k^{-5 / 3} \approx 0.125 C_{T}^{2} k^{-5 / 3} .
$$

However, Wyngaard et al. (1971) reported the relationship between $\Phi_{T}$ and $C_{T}^{2}$ as

$$
\Phi_{T}=0.25 C_{T}^{2} k^{-5 / 3} .
$$

The proportionality coefficient value of 0.25 was justified by the following comment in the references of op. cit.:

"The constant 0.25 stands for $2(2 \pi)^{-1} \Gamma(5 / 3) \sin (\pi / 3)$. Note that we use a range of 0 to $+\infty$ for $k_{1}$, whereas a $-\infty$ to $+\infty$ range is used on p. 25 of Ref 1 ."

where "Ref. 1 " is Tatarskii (1961) and $k_{1}$ is the analog of our $k$.

It appears that this value of 0.25 was an error. Since there is no explicit derivation of Eq. (10) presented by Tatarskii (1961), it seems likely that Wyngaard et al. (1971) misinterpreted the limits of integration used to arrive at Tatarskii's formula [our Eq. (10)] and included the extraneous factor of 2 . However, as follows from our derivation demonstrated above, the adjustment is already made in Eq. (3) to change the range from 0 to $\infty$. This means that the expression (11) from Wyngaard et al. (1971) incorporates a proportionality coefficient that is larger than its actual value by a factor of 2 .

\section{Discussion}

The relationship between $\Phi_{T}$ and $C_{T}^{2}$. in the Wyngaard et al. (1971) form, Eq. (11), has been widely used in structure-parameter calculations (e.g., Kaimal 1973; Asimakopoulos et al. 1976; Wyngaard and LeMone 1980; Moulsley et al. 1981; Kohsiek 1982; Cuijpers and Kohsiek 1989; Beland 1993; Green et al. 1994; Muschinski et al. 2001, 2004; Cheinet and Siebesma 2009; Wilson and 
Fedorovich 2012; Maronga et al. 2013; Maronga 2014; Maronga et al. 2014; Gibbs et al. 2016). The error reported here may affect conclusions regarding the validity of this relationship as compared with observational and numerical data. Specifically, the majority of these cited studies found reasonable agreement between this relationship and various direct methods. Thus, the theoretical shortcomings of the method were implicitly corrected through the use of an improper proportionality coefficient.

Beyond the derivation presented above, we have further reason to believe that Eq. (10) is the correct form of the integral spectral approximation of the temperature structure parameter. In Wyngaard [2010, their Eqs. (15.43) and (16.64), p. 374], a specific form of our Eq. (4) is considered that is subsequently integrated to arrive at an equivalent expression for Eq. (8). Although the approximation (10) is not presented in op. cit., one can easily show that the corresponding coefficient of proportionality is also 0.125 instead of the widely adopted value of 0.25 from Wyngaard et al. (1971). We recommend that Eq. (10) be used going forward.

Acknowledgments. We thank Alan Shapiro and Brian Greene (University of Oklahoma) for helpful conversations related to the presented derivation and Louis Wicker (NOAA National Severe Storms Laboratory) for reviewing the manuscript and offering helpful feedback. Björn Maronga (Leibniz Universität Hannover) provided especially thoughtful comments related to the various methods described in this paper. Funding was provided by NOAA/Office of Oceanic and Atmospheric Research under NOAA-University of Oklahoma Cooperative Agreement NA11OAR4320072, U.S. Department of Commerce.

\section{REFERENCES}

Asimakopoulos, D. N., R. S. Cole, S. J. Caughey, and B. A. Crease, 1976: A quantitative comparison between acoustic sounder returns and the direct measurement of atmospheric temperature fluctuations. Bound.-Layer Meteor., 10, 137-147, https:// doi.org/10.1007/BF00229281.

Beland, R., 1993: Propagation through atmospheric turbulence. The Infrared Electro-Optical Systems Handbook, D. L. Shumaker et al., Eds., SPIE Optical Engineering Press, 159-232.

Cheinet, S., and A. P. Siebesma, 2009: Variability of local structure parameters in the convective boundary layer. J. Atmos. Sci., 66, 1002-1017, https://doi.org/10.1175/2008JAS2790.1.

Cuijpers, J. W. M., and W. Kohsiek, 1989: Vertical profiles of the structure parameter of temperature in the stable, nocturnal boundary layer. Bound.-Layer Meteor., 47, 111-129, https:// doi.org/10.1007/BF00122325.

Essenwanger, O., and E. R. Reiter, 1969: Power spectrum, structure function, vertical wind shear, and turbulence in troposphere and stratosphere. Arch. Meteor. Geophys. Bioklimatol., 18A, 17-24, https://doi.org/10.1007/BF02247861.

Gibbs, J. A., E. Fedorovich, B. Maronga, C. Wainwright, and M. Dröse, 2016: Comparison of direct and spectral methods for evaluation of the temperature structure parameter in numerically simulated convective boundary layer flows. Mon. Wea. Rev., 144, 2205-2214, https://doi.org/10.1175/MWR-D-15-0390.1.

Gradshteyn, I. S., and I. Ryzhik, 2014: Table of Integrals, Series, and Products. 8th ed. Academic Press, 1184 pp.

Green, A. E., K. J. McAneney, and M. S. Astill, 1994: Surface-layer scintillation measurements of daytime sensible heat and momentum fluxes. Bound.-Layer Meteor., 68, 357-373, https:// doi.org/10.1007/BF00706796.

Kaimal, J. C., 1973: Turbulence spectra, length scales and structure parameters in the stable surface layer. Bound.-Layer Meteor., 4, 289-309, https://doi.org/10.1007/BF02265239.

Kohsiek, W., 1982: Measuring CT2, CQ2, and CTQ in the unstable surface layer, and relations to the vertical fluxes of heat and moisture. Bound.-Layer Meteor., 24, 89-107, https://doi.org/ 10.1007/BF00121802.

Kolmogorov, A. N., 1941: The local structure of turbulence in incompressible viscous fluid for very large Reynolds numbers. Dokl. Akad. Nauk SSSR, 30, 301-305.

Maronga, B., 2014: Monin-Obukhov similarity functions for the structure parameters of temperature and humidity in the unstable surface layer: Results from high-resolution large-eddy simulations. J. Atmos. Sci., 71, 716-733, https://doi.org/10.1175/JAS-D-13-0135.1.

, A. F. Moene, D. van Dinther, S. Raasch, F. C. Bosveld, and B. Gioli, 2013: Derivation of structure parameters of temperature and humidity in the convective boundary layer from large-eddy simulations and implications for the interpretation of scintillometer observations. Bound.-Layer Meteor., 148, 1 30, https://doi.org/10.1007/s10546-013-9801-6.

, O. Hartogensis, S. Raasch, and F. Beyrich, 2014: The effect of surface heterogeneity on the structure parameters of temperature and specific humidity: A large-eddy simulation case study for the LITFASS-2003 experiment. Bound.-Layer Meteor., 153, 441-470, https://doi.org/10.1007/s10546-014-9955-x.

Moulsley, T. J., D. N. Asimakopoulos, R. S. Cole, B. A. Crease, and S. J. Caughey, 1981: Measurement of boundary layer structure parameter profiles by acoustic sounding and comparison with direct measurements. Quart. J. Roy. Meteor. Soc., 107, 203-230, https://doi.org/10.1002/qj.49710745113.

Muschinski, A., R. Frehich, M. Jensen, R. Hugo, A. Hoff, F. Eaton, and B. Balsley, 2001: Fine-scale measurements of turbulence in the lower troposphere: An intercomparison between a kite- and balloonborne, and a helicopter-borne measurement system. Bound.-Layer Meteor., 98, 219-250, https://doi.org/10.1023/A:1026520618624.

,$- \ldots$, and B. B. Balsley, 2004: Small-scale and large-scale intermittency in the nocturnal boundary layer and the residual layer. J. Fluid Mech., 515, 319-351, https://doi.org/10.1017/ S0022112004000412.

Tatarskii, V. I., 1961: Wave Propagation in a Turbulent Medium. Dover, 285 pp.

Wilson, C. J., and E. Fedorovich, 2012: Direct evaluation of refractiveindex structure functions from large-eddy simulation output for atmospheric convective boundary layers. Acta Geophys., 60, 1474-1492, https://doi.org/10.2478/s11600-012-0063-3.

Wyngaard, J. C., 2010: Turbulence in the Atmosphere. Cambridge University Press, 393 pp.

, and M. A. LeMone, 1980: Behavior of the refractive index structure parameter in the entraining convective boundary layer. J. Atmos. Sci., 37, 1573-1585, https://doi.org/10.1175/15200469(1980)037<1573:BOTRIS > 2.0.CO;2.

_, Y. Izumi, and S. A. Collins Jr., 1971: Behavior of the refractiveindex-structure parameter near the ground. J. Opt. Soc. Amer., 61, 1646-1650, https://doi.org/10.1364/JOSA.61.001646. 